\title{
Simplified Human Hand Models based on Grasping Analysis
}

\author{
Salvador Cobos, Member, IEEE, Manuel Ferre, Member. IEEE, and Rafael Aracil
}

\begin{abstract}
The purpose of this paper is to analyze in some depth the kinematic behaviour of the human hand, in order to obtain simplified human hand models with the minimum and optimal number of Degrees of Freedom (DoF), and thus achieving an eflicient manipulation task. The statistical analysis is carried out using Principal Components Analysis (PCA). Power and precision grasps are obtained with the use of a Cyberglove and a human hand model with 24 DoF. Finally, these experiments are used to evaluate the best DoF for an appropriate manipulation.
\end{abstract}

\section{INTRODLICTION}

$\mathrm{T}_{\mathrm{h}}$ HE main objective of this work is to use simplified human hand models for cooperative manipulation tasks through human interfaces and teleoperated systems in virtual scenarios.

One difficulty in nnderstanding human hand control is the large number of degrees of freedom (DoF) involved. The high dimensionality of the control space also explains the difficulty on creating effective control algorithms for all human hand models or robotic hands with more than 20 DoF. The purpose of this paper is to determine what DoFs are the most important for controlling a hand model or robotic hand based on grasping analysis. The best simplified hand model depends on the reqnirements of the task, in this case. the degree of realism. the maximum enor produced on the reconstruction and the number of controlled inputs in the hand control performance would have to be considered for a particular task:

The base of all simplified hnman hand models is a kinematic model with 24 DoF that reasonably satisfies realism in simulation [1], [2]. This kinematic model is used for reconstructing simplified hand models with less DoF. The kinematic analysis of the human hand is focused in the role played by the behaviour of the grip in order to decide the more adeqnate simplified hand model for a particnlar manipnlation.

Principal Component Analysis (PCA) has been nsed previously on hand poses such as [3]. [4], and [5]. By means

Manuscript received March 10, 2010. This work was supported in par by the European Commission, as part of the IMMERSENCE project of the Sixth Framework Progranme (IST-4-027141-IP).

Salvador Cobos is with The Group of Robots \& Intelligent Machines of Universidad Politécnica de Madrid, C/José Gutiérez Abascal. 2, 28006,

Madrid, Spain. Phone: (+34) 913363195; fax: (+34) 913363010. e-mail: cobosalvador(â)etsii.upm.es

Manuel Ferre is with The Group of Robots \& Intelligent Machines of Universidad Politánica de Madrid, e-mail: m.fere@upm.es

Rafael Aracil is with The Gronp of Robots \& Intelligent Machines of Universidad Politécnica de Madrid, e-mail: aracilaletsii.upm.es of PCA [6]. an experiment was done with 200 samples in order to obtain five principal components. These samples were obtained using a Cyberglove $(\overrightarrow{\mathrm{R}})$ [7] and a model of the human hand with $24 \mathrm{DoF}$ with objects of different sizes. Therefore, a significant simplification is thus made to the original hand description, as the 24 components are reduced to 5 components.

Cyberglove is also used to read the most important degrees of freedom for reconstructing simplified hand models using inter-finger and intra-finger constraints [8].

Also, this paper is focns in evaluate simplified hand models within a dimensionality reduction [9]. [10]. [11]. [12]. The simplified hand models are evaluated, with regard the hand model of 24 DoF using multi-body dynamics engine.

This paper is organized in the following way: Section II describes briefly the kinematic model of the hnman hand, Section III describes the statistical stndy to analyze the kinematic grasp behaviour, Section IV describes simplified human hand models, and Section V experiments and results. Finally. conclusions are presented in section VI.

\section{KINEMATIC MODEL OF THE HUMAN HAND}

The hand model used for this work is based on the human skeleton. The kinematic model is comprised of 19 links that imitate the corresponding human bones. and 24 degrees of freedom (DoF) that represent the joints. Links and joints are defined by $L_{i, j}$ and $\theta_{i, i^{*}}$ where $i$ represents a finger ( $i=$ Thumb, Index, Middle, Ring or Little) and $j$ its comesponding link or joint respectively. Two kinematic configurations are considered in this hand model, one for the thumb and another for the rest of the fingers. Therefore, the same kinematic configuration is used for the index, middle. ring and little fingers. This configuration is defined by 5 joints and 4 links: metacarpal $\left(L_{i, M e}\right)$, proximal $\left(L_{i, P}\right)$, middle $\left(L_{i, M i}\right)$ and distal $\left(L_{i, D}\right)$ links. The joints are defined as: carpometacarpal $\left(\theta_{i, C M C}\right)$, proximal interphalangeal $\left(\theta_{1, P I P}\right)$ distal interphalangeal $\left(\theta_{3, D I P}\right)$ and metacarpophalangeal, which is modelled by a universal joint ( $2 \mathrm{DoF}$ ) that defines the abduction/adduction ( $\theta_{i, M C P_{-}}$) and flexion/extension $\left(\theta_{i, M C P} f_{e}\right)$ rotations. The thumb is modelled by $4 \mathrm{DoF}$ and 3 links: metacarpal $\left(L_{T, M}\right)$, proximal $\left(L_{T, P}\right)$, and distal $\left(L_{T, D}\right)$. The thumb joints are defined as: metacarpophalangeal $\left(\theta_{T, M C P, f e}\right)$, interphalangeal $\left(\theta_{T, I P}\right)$ and trapezionetacarpal, which is also defined by a universal joint that defines the abduction/adduction $\left(\theta_{T_{r} M C_{-} a a}\right)$ and flexion/extension $\left(\theta_{T, T M C f_{e}}\right)$ respectively. The rest of the joints are modelled by revolute joints. Forward kinematics and inverse kinematics are described in more detail in S. Cobos et al. [3]. 


\section{PCA ANALYSIS}

The PCA analysis was carried out nsing a data base from 200 samples. These samples were obtained through Cyberglove and hand model with $24 \mathrm{DoF}$, thns having a collection of vinual gestures by means of handling real objects. The type of grasps produced are: prismatic precision, prismatic power. circular power and circular precision (fignres 1.a to l.e), with objects of different sizes. This procedure performs a principal components analysis nsing the covariance matrix. The pnrpose of the analysis is to obtain a small number of linear combinations of the 24 variables which account for most of the variability in the data. In this case, 5 components are extracted as requested. Together, the 3 Principal Components acconnt for $\sim 88.4 \%$ of the variability in the original data. Therefore, these functions represent a new compressed 5-dimension of the 24 DoF that have been nsed to model the hand gestnre.

The purose of this analysis is to identify if it is feasible reducing the degrees of freedom and to identify the most imponant degrees of freedom for the reconstruction of a specific grasp (power or precision). In other words, how many fingers and DoF are required for the reconstruction of the gestures according to Cntkosky classification [13]?

By means of PCA. it is possible to identify how many variables are required to represent the information of the 24 $\mathrm{DoF}$. Therefore. PCA was used to identify the effective degrees of freedom more precisely.

TABLE I

DEGREES CF FREEDOM MOST IMPORTANT FOR POWER AND PRECISION GRASP FROM THE PRINCIPAL CCMPCNENT ANALYSIS

\begin{tabular}{|c|c|c|}
\hline Finger & Joint & Symbol \\
\hline Thumb & $\begin{array}{l}\text { Trapezionetacarpal } \\
\text { abduction:adduction }\end{array}$ & $\theta_{\text {T.TMK_a }}$ \\
\hline Thuinb & $\begin{array}{l}\text { Trapezionetacarpal } \\
\text { flexion extension }\end{array}$ & $\theta_{T, T M K, A}$ \\
\hline Index & $\begin{array}{l}\text { Metacarpoplalangeal } \\
\text { abduction/adduction }\end{array}$ & $\theta_{\text {d,MCP_an }}$ \\
\hline Index & Proximal Interphalangea] & $\theta_{d, F F P}$ \\
\hline Middle & $\begin{array}{l}\text { Metacarpoplalangeal } \\
\text { flexion/extension }\end{array}$ & $\theta_{M M C P F}$ \\
\hline Middle & Proximal Interphalangea] & $\theta_{\text {MYPIP }}$ \\
\hline Ring & $\begin{array}{l}\text { Metacarpophalangeal } \\
\text { flexion/extension }\end{array}$ & $\theta_{R, A C P}$ \\
\hline Ring & Proximal Interphalangeal & $\theta_{R, P I P}$ \\
\hline Little & $\begin{array}{l}\text { Metacarpophalangeal } \\
\text { flexion extension }\end{array}$ & $\theta_{L, M C P, S}$ \\
\hline Little & Proximal Interphalangeal & $\theta_{L P J P}$ \\
\hline
\end{tabular}

The kinematic analysis of hand postures for grasping objects showed that a considerable reduction of the degrees of freedom is permissible. In particular, the 5 principal components demonstrate that $88.4 \%$ of the variance can describe the five principal gestnres considered in this analysis. In order to obtain the most relevant degrees of freedom, the weight component, and standard components (rescaled components) of each component are used. The 10 degrees of freedom most important are obtained by applying a weighted standardization over the weight component, and standard components. Table I and figure 2 show the degrees of freedom most imponant. Therefore. one interpretation of these findings in terms of gesture reconstruction is that one flexion of all fingers and the abduction/adduction for thumb and index fingers mnst be considered important variables. Fignre 2 shows in red colour the most imporlant degrees of freedom and the less important degrees of freedom in blue colonr.

On the other hand. the least significant degrees of freedom are the carpometacarpal (CMC) joints. Comparing the weights between the four $\mathrm{CMC}$. the most imponant $\mathrm{CMC}$ joint is the $\mathrm{CMC}$ of the Little finger $\left(\theta_{L, C M C}\right)$.

Flexion is strongly related in all fingers. Therefore, the chosen flexion conld be $\theta_{1, H C P \text { fe }}, \theta_{i, P I P}$, or $\theta_{1, D I P}$ joints for index. middle, ring and little fingers and $\theta_{T, T M C f e}, \theta_{T, M C P}$ fe. or $\theta_{T, I P}$ for the thumb. The results of the analysis show that the middle finger, the ring finger and the little finger have 2 very relevant flexions. The possibility to choose among any of the 2 flexions for these fingers is given by the strong relation that exists among the 3 flexions. Any of these flexions can be represented as a lineal combination of the other 2 flexions. Finally, the analysis shows that the 3 most imponant abductions are: $\theta_{T, T M C_{-} \alpha u}, \theta_{I, M C P_{-} a u}$ and $\theta_{L_{M} M C_{-} a u}$.

\section{SIMPLIFIEd Human HaNd MODElS}

This section describes simplified human hand models that properly represent the kinematic behavionr of the human hand in accordance with the precision and application required. The human hand model of $24 \mathrm{DoF}$ is used as a basis for comparison among simplified hand models with fewer degrees of freedom than the $24 \mathrm{DoF}$ of the hand model described in section 2 .

Kinematic constraints are used in order to obtain simplified hand models (SHM), which allow redncing the nnmber of independent variables or joints in the original model. In other words. with few independent variables it is possible to reconstruct a gesture of 24 degrees of freedom with an acceptable error with respect to the original gestnre of 24 degrees of freedom not reconstructed. Simplified hnman hand models are obtained nsing dependent and independent variables; these dependent variables or dependent joints are calculated using kinematic constraints.

The reduction of elements from 13 to 1 DoF leads to increasingly rely on interpolations and constraints associated with an increased error in the grip trajectory when the dependent variables are obtained. 


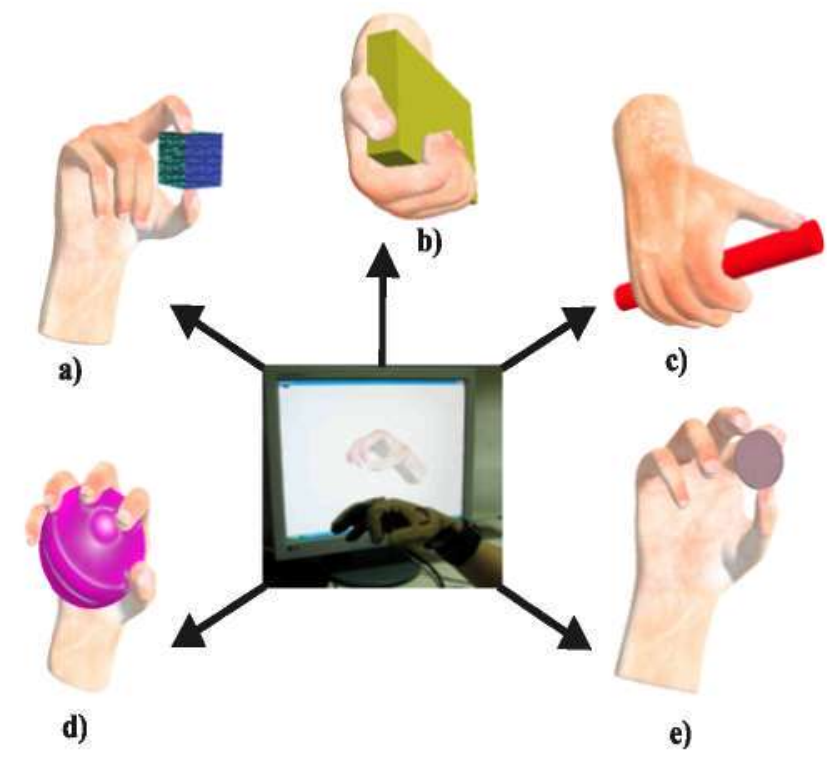

Fig. 1. Grasp rendering of various objects using a Cyberglove and a hand model with $24 \mathrm{DoF}$. a) Prismatic Precision, b) Heavy Power, c) Prismatic Power, d) Circular Power and e) Circular Precision.

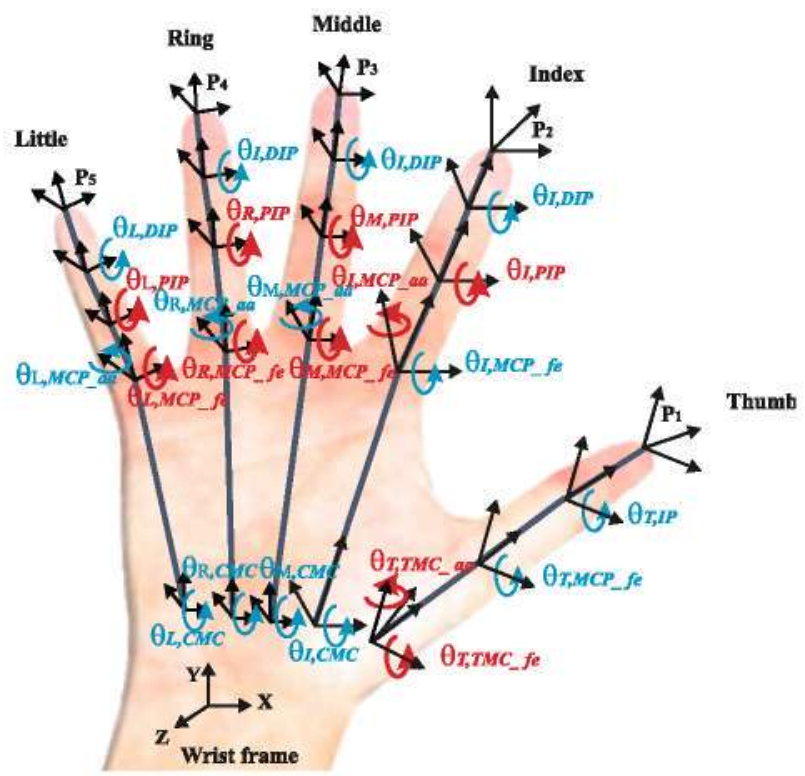

Fig. 2. Degrees of freedom most important obtained with principal component analysis.

Somehow, this technique depends on optimizing the functionality of a particular inter-finger or intra-finger constraint.

Many manipulations involve similar movements among fingers, e.g., a gesture done with the information of five fingers can be simplified by using only the information provided for three fingers. In this case, these three fingers can be thumb, index and ring, creating the same movement for the middle finger through the information of the index and the little finger through the information of the ring finger.
The simplified hand models should be used depending on the relation between the number of degrees of freedom and the allowed error in the application.

The degree of dexterity that can be achieved depends mainly on the largest number of independent variables having thumb and index finger inside the SHM. The abduction/adduction of the thumb and index finger is very important because at least one degree of freedom from these fingers is considered as an independent variable in all the simplified hand models, thus the flexion of the proximal interphalangeal (PIP) joint of the index finger is included in all the simplified hand models.

The abduction/adduction of the thumb Trapeziometacarpal (TMC) joint is important because with a flexion of the interphalangeal (IP) joint can produce the opposition of the thumb with the other fingers. In summary the universal joints of the thumb and index fingers are very important for obtaining simplified hand models because of the information they provide.

Finally, SHM have been used for manipulated power and precision grasps in a virtual environment. The scenario has been performed integrated a collision and dynamics libraries.

\section{A. Simplified hand models from 1 to 6 degrees of freedom}

To control a gesture with one degree of freedom has been demonstrated previously in a robotic hand e.g. The Tuat/Karlsruhe Hand [14]. This hand is designed with 24 joints and 1 DoF controlled, and it is able to do circular power grasps.

In general, models from 1 to $6 \mathrm{DoF}$ are appropriate for power grasps with security and stability in the grip without achieving a great precision and skill in handling for precision grasps. In this category the models from 1 to 3 are capable of performing circular power grasps and models from 4 to 6 are capable of performing circular and prismatic power grasps.

The most important flexion was the PIP joint, as resulted in the PCA analysis. The reading or extraction of the information provided from this joint is only possible with a Cyberglove. For a haptic application this is not feasible, because in order to read some degree of freedom of a human hand, it can be done with the fingertips. For this reason, for a haptic application [15], [16] the distal interphalangeal (DIP) joints and the IP joint are ideal for independent joints.

Table II shows the degrees of freedom used to perform a manipulation for circular power grasps. Figures $3 a$ ) and $3 b$ ) show two examples of SHM with 3 and 6 DoF respectively for a Cyberglove interface.

The Barret Hand [17] is a good example to compare SHM with $4 \mathrm{DoF}$ from the thumb finger, index finger and little finger. The barret hand is composed by three fingers, four servomotors and 7 joints. Both hands are used to do power handling tasks. 
TABLE II

DEGREES OF FREEDOM FOR SHM FROM 1 TO 6 DOF

\begin{tabular}{ll}
\hline \hline DoF & \multicolumn{1}{c}{ Independent Joints } \\
\hline 1 & $\theta_{I, P I P}$ \\
2 & $\theta_{I, P I P}, \theta_{T, T M C_{-} a a}$ \\
3 & $\theta_{I, P I P}, \theta_{T, T M C_{\_} a a}, \theta_{T, T M C_{f e}}$ \\
4 & $\theta_{I, P I P}, \theta_{T, T M C_{-} a a}, \theta_{T, T M C_{f e},}, \theta_{l, M C P_{a} a a}$ \\
5 & $\theta_{I, P I P}, \theta_{T, T M C_{-} a a}, \theta_{T, T M C_{f} f}, \theta_{l, M C P \_a a}, \theta_{M, P I P}$ \\
6 & $\theta_{I, P I P}, \theta_{T, T M C_{a} a}, \theta_{T, T M C \text { fe }}, \theta_{I, M C P \text { aa }}, \theta_{M, P I P}, \theta_{L, P I P}$ \\
\hline \hline
\end{tabular}

\section{B. Simplified hand models from 9 to 14 degrees of freedom}

Greater precision and dexterity is derived from 9 degrees of freedom, thus it is possible to carry out precision grasps. Simplified hand models of 9 to $14 \mathrm{DoF}$ are more precise for both types of grasp: precision and power grasps.

These SHM models are capable of performing the four types of grasps: circular power, prismatic power, circular precision and prismatic precision. Table III shows the degrees of freedom for reconstructing models from 9 to $14 \mathrm{DoF}$. Figures 4a) and 4b) show two examples of SHM with 9 and 14 DoF respectively for a Cyberglove interface.

In this classification, four robotic hands can be compared with SHM of $9 \mathrm{DoF}, 11 \mathrm{DoF}, 13 \mathrm{DoF}$ and $14 \mathrm{DoF}$. The Stanford/JPL Hand [18] is composed by three fingers, and 9 DoF controlled. The Okada hand [19] has 11 DoF controlled. The DLR hand [20], [21] is composed by four fingers, and 13 DoF controlled. The Robonaut hand [22] is composed by five fingers, and $14 \mathrm{DoF}$ controlled. These hands are used to do precision handling tasks.

TABLE III

DEGREES OF FREEDOM FOR SHM FROM 9 TO 14 DOF

\begin{tabular}{|c|c|}
\hline DoF & Independent Joints \\
\hline 9 & $\begin{array}{l}\theta_{I, P I P}, \theta_{T, T M C_{\_} a a}, \theta_{T, T M C, f e}, \theta_{l, M C P \_a a}, \theta_{M, P I P}, \theta_{L, P I P}, \\
\theta_{R, P I P}, \theta_{L, M C P \_a a}, \theta_{L, C M C}\end{array}$ \\
\hline $\begin{array}{l}10 \\
11 \\
12 \\
13 \\
14\end{array}$ & $\begin{array}{l}\text { SHM } 9 \text { DoF }+\theta_{I, M C P} \text { fe } \\
\text { SHM } 10 \mathrm{DoF}+\theta_{T, M C P} \text { fe } \\
\text { SHM } 11 \mathrm{DoF}+\theta_{M, M C P} \text { fe } \\
\text { SHM } 12 \mathrm{DoF}+\theta_{R, M C P} \\
\text { SHM } 13 \mathrm{DoF}+\theta_{L, M C P} \text { fe }\end{array}$ \\
\hline
\end{tabular}

\section{Simplified hand models from 15 to 24 degrees of freedom}

A higher level of realism and sensitivity is achieved with models from 15 to $24 \mathrm{DoF}$. Only the model with $24 \mathrm{DoF}$ is capable of performing simulation with arc palm deformation.

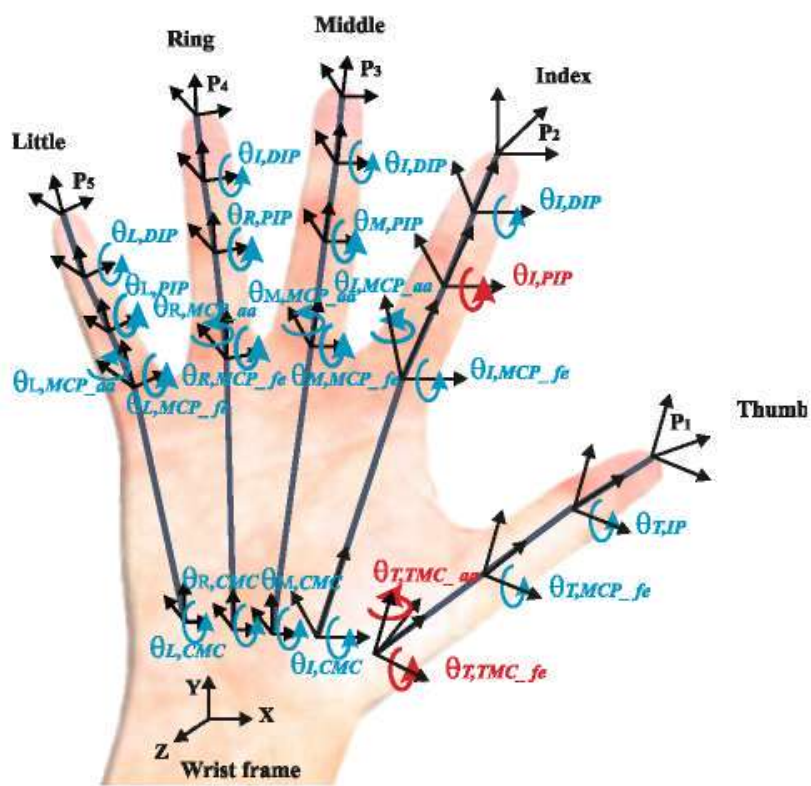

a)

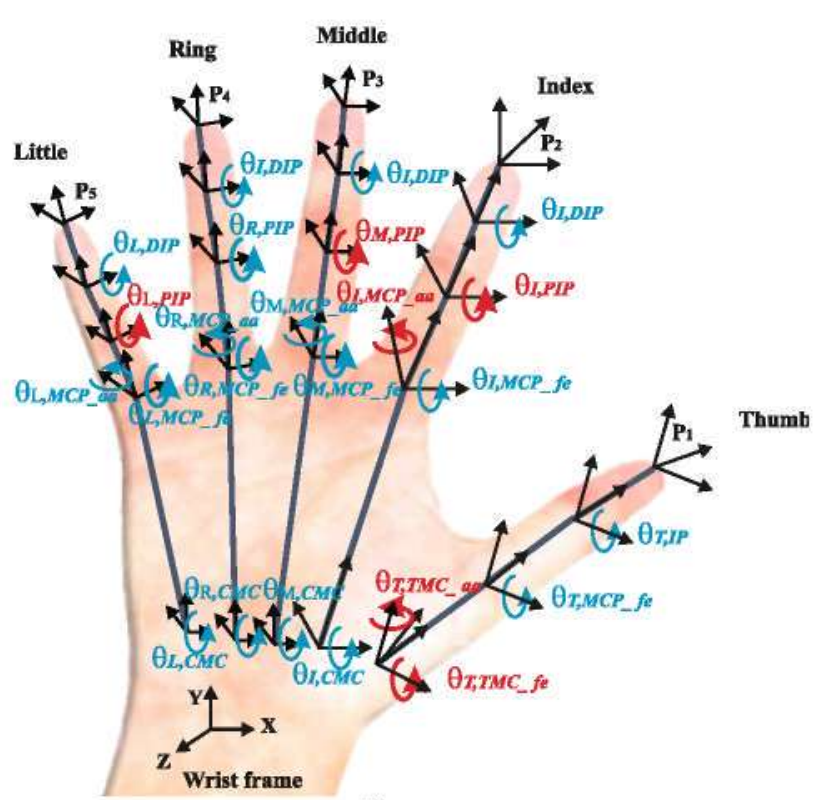

b)

Fig. 3. Simplified hand model for power grasps a) Simplified hand model with 3 DoF b) Simplified hand model with 6 DoF.

With 15,16 and 17 DoF is possible to have the 3 important flexions for index, thumb and middle fingers. All this information helps carrying out precision grasps with great realism. Table IV shows the degrees of freedom for reconstructing models from 15 to $24 \mathrm{DoF}$.

These models serve for applications that require a greater realism, sensitivity in handling or description of a human hand gesture. The integration of these models in a haptic application should be computationally expensive, depending on the task that it is required to manipulate and the amount of data for calculating in real time. 

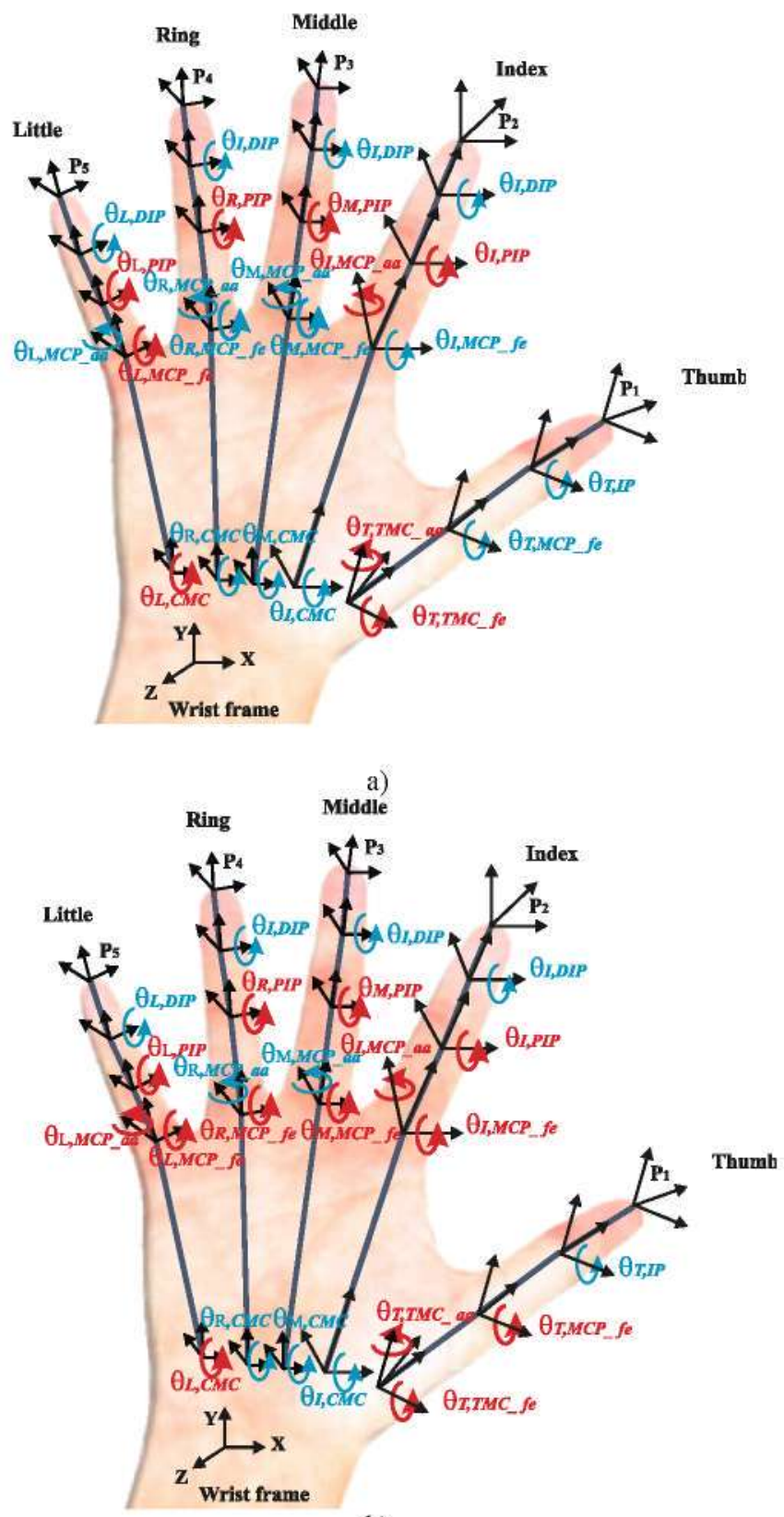

b)

Fig.4. Simplified hand models for Power and Precision grasps. a) Simplified hand model with $9 \mathrm{DoF}$ b) Simplified hand model with 14 DoF.

Finally, in this classification the SHM of 16 DoF and 24 DoF can be compared with two robotic hands. The Gifu Hand [23] is composed by five fingers, and $16 \mathrm{DoF}$ controlled. The Shadow Dexterous Hand [24] is driven by 40 Air Muscles mounted on the forearm with 22 DOF for the fingers and $2 \mathrm{DoF}$ for the wrist. These hands are used to do dexterous handling tasks.

\section{EXPERIMENTS WITH SIMPLIFIED HAND MODELS}

Simplified hand models are developed with regards to the type of manipulation task, this manipulation characteristic depends on the trajectory carried out by the joints inside of two movement spaces: circular and prismatic. Therefore, the SHM are evaluated in these two types of trajectories.

This section presents some experiments carried out with simplified hand models. SHM are evaluated by using multibody dynamics software in order to manipulate virtual objects for force grasp and dexterity grasp, it involves grasping a circular or prismatic object, and retain a stable handling. The collision detection and computation times are measured for: 24 DoF hand model, SHM with 9 DoF and SHM with 6 DoF. The multi-body dynamics software used for this evaluation is: Chrono Engine Library [25]. All experiments were executing in an Intel Core 2 Quad CPU at $2.40 \mathrm{GHz}$ under windows XP operative system.

Figures 5 and 6 contain a comparative graph among the computation times of each SHM. The data collection is obtained through 250 samples for circular and prismatic grasps. Each scenario was replicated 50 times so as to get a representative measurement of time. The following figures contain a comparative graph among the computation times of each SHM. A significant reduction on time calculation for collision detection between SHM with $24 \mathrm{DoF}$ and SHM with $9 \mathrm{DoF}$ such as shown in figure 5 can be observed.

Figure 6 shows the time used for collision detection in 24 DoF hand model, SHM with 9 DoF and SHM with 6 DoF for handling circular grasps. The SHM with 6 DoF was used for evaluating circular grasp handling. In figure 6 , the difference in time that exists among 3 hand models can be seen. In this evaluation, prismatic grasp handling was evaluated between 24 DoF hand model and 9 DoF SHM because the 6 DoF SHM is develop just for circular grasp.

TABLE IV

DEGREES OF FREEDOM FOR SHM FROM 15 TO 24 DOF

\begin{tabular}{|c|c|}
\hline DoF & Independent Joints \\
\hline 15 & $S H M 14 D o F+\theta_{I, D I P}$ \\
\hline 16 & $S H M 15 D o F+\theta_{T, I P}$ \\
\hline 17 & $S H M 16 D o F+\theta_{M, D I P}$ \\
\hline 18 & $S H M 17 D o F+\theta_{R, D I P}$ \\
\hline 19 & $S H M 18 D o F+\theta_{L, D I P}$ \\
\hline 20 & SHM $19 \mathrm{DoF}+\theta_{R, M C P} a a$ \\
\hline 24 & $S H M 20 D o F+\left(\theta_{R, C M C}, \theta_{M, C M C}, \theta_{I, C M C}, \theta_{M, M C P \_a a}\right)$ \\
\hline
\end{tabular}

\section{CONCLUSION}

This paper presented simplified human hand models based on an important statistical analysis such as principal components. This study serves to determine what variables are the most important to take into account for two big spaces of grasping: power and precision grasps. The human role on grasping is considering by means of the abstraction of the human hand joints using a Cyberglove. These models with Number of DoF $<24$ DoF are useful for diverse applications that require achieving an efficient handling 
depending on the level of dexterity, stability, computational cost and realism required in handling tasks.

\section{Prismatic Manipulation}

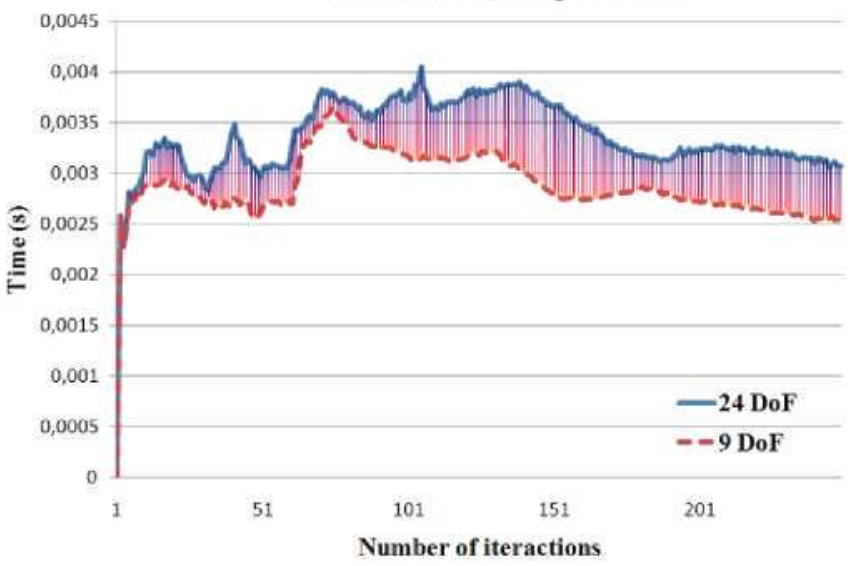

Fig. 5. Time used for collision detection in $24 \mathrm{DoF}$ hand model and SHM with 9 DoF for a prismatic grasp handling

\section{Circular Manipulation}

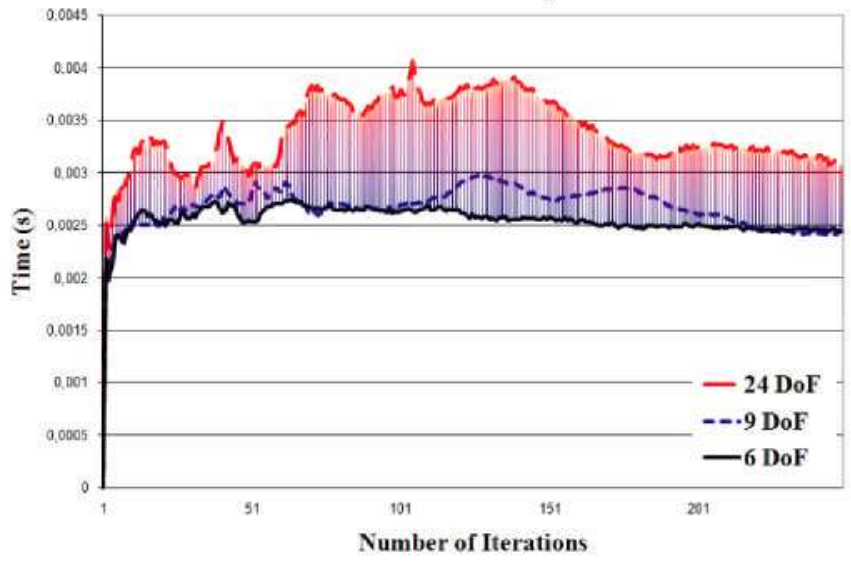

Fig. 6. Time used for collision detection among $24 \mathrm{DoF}$ hand model, SHM with $9 \mathrm{DoF}$ and SHM with $6 \mathrm{DoF}$ for circular grasp handling.

\section{ACKNOWLEDGMENT}

Many thanks to Conacyt and UPM by the supports offered to Salvador Cobos during your Ph.D. Also, thanks to Victor Eslava in your help to perform the experiments in section V.

\section{REFERENCES}

[1] S. Cobos, M. Ferre, M.A. Sánchez-Urán, J. Ortego and C. Peña. Efficient Human Hand Kinematics for manipulation Tasks, IEEE/RSJ International conference on Intelligent Robots and Systems, pp. 2246 2250. IROS, 2008.

[2] S. Cobos, M. Ferre, M.A. Sánchez -Urán and J. Ortego. Simplified Hand Configuration for Object Manipulation, In Proc. Eurohaptics, pp. 730-735. Eurohaptics, 2008.

[3] S. Cobos, M. Ferre, M. A. Sánchez-Urán, J. Ortego and R. Aracil. Human hand descriptions and gesture recognition for object manipulation. Computer Methods in Biomechanics and Biomedical Engineering, Vol. 13, No. 3, pp. 305 - 317.
[4] Y. Endo, S. Kanai, T. Kishinami, N. Miyata, M. Kouchi, and M. Mochimaru, A computer-aided ergonomic assessment and product design system using digital hands, Digital Human Modeling, HCII 2007, V.G. Duffy (Ed.): LNCS 4561, pp. 833-842, 2007

[5] M. Santello, M. Flanders, and J. F. Soechting. Postural Hand Synergies for Tool Use. The Journal of Neuroscience, December 1 , 1998, 18(23):10105-10115.

[6] I. T. Jollife. Principal Component Analysis, Second edition. Springer 2002.

[7] http://www.cyberglovesystems.com

[8] S. Cobos, M. Ferre, J. Ortego, and M.A. Sánchez -Urán. Constraints for Realistic Hand Manipulation. The $10^{\text {th }}$ annual International Workshop on Presence, PRESENCE 2007, pp. 369-370

[9] M.T. Ciocarlie and P. K. Allen. Hand Posture Subspaces for Dexterous robotic Grasping. The International Journal of Robotics Research, July 1, 2009, 28(7):851 - 867 .

[10] M.T. Ciocarlie, C. Goldfeder, P. Allen, Dimensionality reduction for hand-independent dexterous robotic grasping. Proc. in 2007 IEEE/RSJ International Conference on Intelligent Robots and Systems San Diego, CA, USA, Oct 29 - Nov 2, 2007 pp. 3270- 3275

[11] N. Miyata, M. Kouchi, T. Kurihara, M. Mochimaru, Modeling of Human Hand Link Structure from Optical Motion Capture Data, IEEE/RSJ Int. Conf. Intelligent Robots and Systems, pp. 2129-2135, 2004.

[12] Y. Endo, S. Kanai, N. Miyata, M. Kouchi and M. Mochimaru, An Application of a Digital Hand to Ergonomic Assessment of Handheld Information Appliances. In: Proc. of SAE 2006 Digital Human Modeling for Design and Engineering Conference, 2006-01-2325 (2006)

[13] M.R. Cutkosky. On grasp choice, grasp models, and the design of hands for manufacturing tasks. IEEE trans. Robotics and automation, pp. $269-279,1989$

[14] N. Fukuya, S. Toyama, T. Asfour and R. Dillmann. Design of the TUAT/Karlsruhe Humanoid Hand. In Proc. IEEE/RSJ International Conference on Intelligent Robots and Systems, (2000), pp. 1754. 1759.

[15] M. Oyarzabal, M. Ferre, S. Cobos. M. Monrroy, J. Barrio and J. Ortego. Multifinger Haptic Interface for Collaborative Tasks in Virtual Environments. In Proc. LNCS, pp. 673-680, 2007.

[16] M. Monroy, M. Oyarzabal, M. Ferre, A. Campos, and J. Barrio, MasterFinger: Multi-finger Haptic Interface for Collaborative Environments, EuroHaptics 2008, LNCS 5024, pp. 411-419, 2008.

[17] W.T. Townsend. MCB-Industrial robot feature article- Barrett Hand grasped, in industrial Robot: An international Journal Vol. 27 No. 3 pp. 181-188.

[18] K.S. Salisbury and B. Roth. Kinematics and force analysis of articulated mechanical hands, Journal of Mechanisms, Transmissions and Actuation in Design, 105 , pp. $35-41$.

[19] T. Okada (1982). Computer control of multijointed finger system for precise object handing, IEEE Transactions on systems, Man and Cybernetics, Vol. Smc-12, No. 3, pp. 289-299

[20] J. Butterfass, M. Grebenstein, H. Liu and G. Hirzinger. DLR-Hand II: Next Generation of a Dextrous Robot Hand, Proc. IEEE Int. Conf. On Robotics and Automation, pp. 907-912.

[21] J. Butterfass, G. Hirzinger, S. Knoch and H. Liu. DLR's Multisensory articulated Part I: Hard- and Software Architecture, in Proc. IEEE International Conference on Robotics and Automation, pp. 20812086.

[22] C.S. Lovchik and M.A. Diftler. The Robonaut Hand: a Dexterous Robot Hand for Space, Proc. IEEE International conference on Robotics and Automation, pp. 907-912.

[23] H. Kawasaki, H. Shimomura, Y. Shimizu, Educational-industrial complex development of an anthropomorphic robot hand "Gifu Hand", Advanced Robotics, Vol. 15, No. 3 pp. 357-367

[24] http://www.shadowrobot.com

[25] http://www.deltaknowledge.com/chronoengine/ 